\title{
Exosomes and breast cancer drug resistance
}

\author{
Xingli Dong ${ }^{1,2}$, Xupeng Bai $\mathbb{C}^{2,3}$, Jie $\mathrm{Ni}^{2,3}$, Hao Zhang $\mathbb{D}^{4}$, Wei Duan ${ }^{5}$, Peter Graham ${ }^{2,3}$ and Yong Li $\mathbb{D}^{2,3,6}$
}

\begin{abstract}
Drug resistance is a daunting challenge in the treatment of breast cancer (BC). Exosomes, as intercellular communicative vectors in the tumor microenvironment, play an important role in BC progression. With the in-depth understanding of tumor heterogeneity, an emerging role of exosomes in drug resistance has attracted extensive attention. The functional proteins or non-coding RNAs contained in exosomes secreted from tumor and stromal cells mediate drug resistance by regulating drug efflux and metabolism, pro-survival signaling, epithelial-mesenchymal transition, stem-like property, and tumor microenvironmental remodeling. In this review, we summarize the underlying associations between exosomes and drug resistance of $\mathrm{BC}$ and discuss the unique biogenesis of exosomes, the change of exosome cargo, and the pattern of release by BC cells in response to drug treatment. Moreover, we propose exosome as a candidate biomarker in predicting and monitoring the therapeutic drug response of $\mathrm{BC}$ and as a potential target or carrier to reverse the drug resistance of $\mathrm{BC}$.
\end{abstract}

\section{Facts}

- Tumor-derived exosomes mediate the chemoresistance of $\mathrm{BC}$ by reducing the intracellular accumulation of chemotherapeutic drugs and delivering functional cargos that activate pro-survival signaling and unchecked cell cycle progression.

- Tumor-derived exosomes mediate the hormonal resistance of $\mathrm{BC}$ by transferring functional cargos that upregulate $\mathrm{ER} \alpha$ expression and hormone-independent signaling.

- Tumor-derived exosomes mediate HER2-targeted drug resistance of $\mathrm{BC}$ by direct interaction of HER2overexpressing exosomes with targeted drugs or by transcriptome reprogramming of the HER2 ${ }^{+} \mathrm{BC}$ into a HER2-independent pattern.

- Tumor- and TME-derived exosomes carrying immunosuppressive molecules mediate the immunotherapeutic resistance of $\mathrm{BC}$ by remodeling the tumor immune microenvironment.

Correspondence: Yong Li (y.li@unsw.edu.au)

'Department of Biopharmaceutical Sciences, College of Pharmacy,

Harbin Medical University, 150081 Harbin, Heilongjiang, China

${ }^{2}$ St George and Sutherland Clinical School, Faculty of Medicine, UNSW Sydney,

Kensington, NSW 2052, Australia

Full list of author information is available at the end of the article

Edited by F. Pentimalli
- Tumor-derived exosomes mediate enhanced EMT and stem-like property of drug-resistant BC.

- TME-derived exosomes mediate the tumor microenvironmental remodeling that favors the drug resistance of $\mathrm{BC}$.

- The exosome is proposed as a candidate biomarker in predicting and monitoring the therapeutic drug response of $\mathrm{BC}$.

- The exosome is proposed as a potential therapeutic target or carrier to reverse drug resistance.

\section{Open questions}

- How do exosomes mediate drug resistance in $\mathrm{BC}$ ?

- By what mechanisms do exosomes promote BC progression?

- What is the role of exosomes in mediating the phenotypic transformation of $\mathrm{BC}$, such as enhanced stemness and EMT?

- How do exosomes induce tumor microenvironmental remodeling to facilitate $\mathrm{BC}$ drug resistance?

- Do exosomes correlate with immunotherapeutic resistance in $\mathrm{BC}$ ?

- How can exosomes be utilized as a biomarker to predict and monitor the therapeutic drug response in $\mathrm{BC}$ patients? 
- What are the necessary steps that will lead to the clinical applicability of the exosomes as biomarkers?

- How can exosomes be applied as a target to overcome drug resistance in $\mathrm{BC}$ ?

- How can exosomes be applied as a drug carrier in the context of drug resistance in $\mathrm{BC}$ ?

\section{Introduction}

Breast cancer $(\mathrm{BC})$ is the most commonly diagnosed cancer and the leading cause of cancer-related death in females worldwide ${ }^{1}$. According to the Globocan statistics, there were about 2.1 million newly diagnosed female BC cases in 2018, accounting for approximately $25 \%$ of cancer cases in women. Clinically, conventional drugs, such as chemotherapeutic, hormonal, and targeted drugs ${ }^{2}$, remain the first-line drug treatment. Several immunotherapeutic drugs demonstrated promising efficacy in clinical trials ${ }^{3}$. Nevertheless, the treatment outcome is often restricted by drug resistance, as well as the lack of biomarkers for predicting the treatment response. Therefore, further understanding the potential molecular mechanisms responsible for drug resistance and seeking reliable biomarkers to predict and monitor the therapeutic response are necessary for a more effective $\mathrm{BC}$ treatment.

Over the past decades, mounting studies demonstrated that the tumor microenvironment (TME) is a major determinant not only of tumor growth, progression, and metastasis but also of treatment resistance ${ }^{4-8}$. Extracellular vesicles (EVs) are heterogeneous populations of small membrane vesicles that are released by cells, carrying a variety of functional nucleic acids, proteins, and lipid cargos important in cell-cell communication ${ }^{9-12}$. According to the size and source, EVs are classified into exosomes $(50-100 \mathrm{~nm} \text { in diameter; endosomal origin) })^{13}$, ectosomes $(100-1000 \mathrm{~nm}$ in diameter; direct budding of the plasma membrane, also known as microparticles/ microvesicles $)^{14}$, apoptotic bodies $(1-5 \mu \mathrm{m} \text { in diameter })^{15}$, large oncosomes $(1-10 \mu \mathrm{m})^{9}$, and other miscellaneous EV subsets $^{13}$. Exosomes are of particular interest in biological research because their unique biogenesis involves distinct intracellular regulatory processes that give them specific cargos and thus different biological functions ${ }^{14-16}$. Exosomes transfer these cargos to recipient cells and induce their phenotypic changes. Increasing evidence indicates that exosome-mediated cell communication is a newly identified mechanism underlying drug resistance ${ }^{11,17}$. By directly exporting drugs, inducing drug inactivation, and delivering functional proteins and non-coding RNAs, exosomes contribute significantly to $\mathrm{BC}$ drug resistance $^{18,19}$.

$\mathrm{BC}$ is a highly heterogeneous disease comprised of multiple distinct subtypes, which are different in genetics, pathology, and clinic behaviour, while exosomes mediate drug resistance through different mechanisms in different subtypes of $B C$, suggesting an extensive role of exosomes in $\mathrm{BC}$ progression. In this review, we summarize the latest progress on the association of exosomal cargos with $\mathrm{BC}$ drug resistance (Table 1). We also discuss the potential role of exosomes as cancer biomarkers to reflect TME changes and to predict therapeutic responses, as well as their potential therapeutic applications against antitumor drug resistance.

\section{Exosome and chemoresistance in BC}

Chemotherapy is a standard regime for invasive $\mathrm{BC}$, especially triple-negative breast cancer (TNBC). However, $\mathrm{BC}$ cells can employ a variety of mechanisms to escape cell death induced by chemotherapeutic drugs. These mechanisms mainly include the efflux and inactivation of drugs ${ }^{20}$, activation of bypass signaling or prosurvival pathways, enhancement of DNA damage repair ${ }^{21}$, and induction of epithelial-mesenchymal transition $(\mathrm{EMT})^{22}$ and stem-like property ${ }^{23}$. Also, a large body of evidence demonstrated that the content of exosomes secreted by tumor cells changes in response to cellular stress induced by anticancer therapy, resulting in the transfer of drug-resistant phenotypes among tumor cells in $\mathrm{BC}^{24-26}$. The exosome-mediated transfer of chemotherapeutic resistance between resistant and sensitive BC cells is shown in Fig. 1.

\section{Exosomes-mediated drug efflux and inactivation}

The sufficient intracellular accumulation of cytotoxic drugs in cancer cells is the prerequisite for the efficacy; thus increased drug efflux is an important action causing chemoresistance. To date, at least three mechanisms of increased chemotherapeutic drug export are known to be mediated by exosomes. The first is exosome-mediated direct drug efflux (Fig. 1a). As early as 2003, a positive correlation between EV shedding-related gene and drug resistance in cancer cell lines was described ${ }^{27}$. They found that $\mathrm{BC}$ cells could export the chemotherapeutic drug doxorubicin (DOX) into the extracellular medium through vesicle formation ${ }^{27}$. The second mechanism is the exosome-mediated horizontal transfer of membraneembedded drug efflux pumps to sensitive cancer cells (Fig. 1b). Adenosine triphosphate (ATP)-binding cassette $(\mathrm{ABC})$ transporter utilizes ATP to efflux various xenobiotics, including anticancer drugs with different structures and properties ${ }^{28,29}$. The ABC subfamily B member 1 gene encodes for the drug transporter, permeability glycoprotein $(\mathrm{P}-\mathrm{gp})^{30}$. There are a large number of studies showing that P-gp can be transferred from drug-resistant tumor cells to sensitive cells through exosomes, leading to acquired drug resistance in vitro and in vivo ${ }^{31-33}$. Similarly, Lv et al. ${ }^{24}$ demonstrated that P-gp was transported via exosomes from docetaxel (DOC)-resistant $\mathrm{BC}$ cells to DOC-sensitive cells, leading to an acquired DOC 


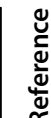

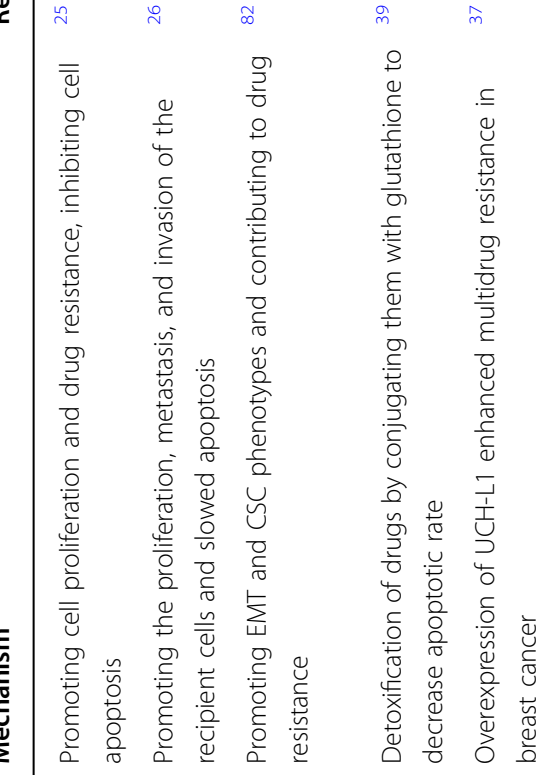

q

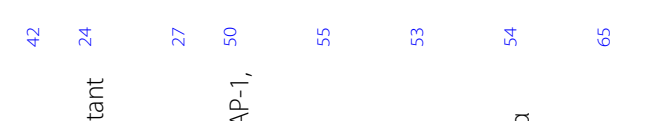

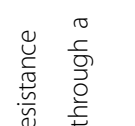

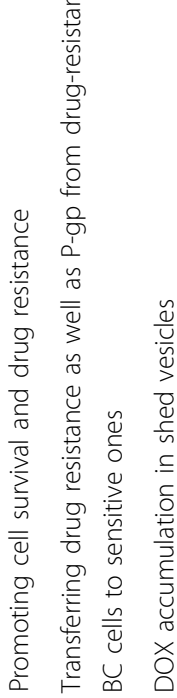

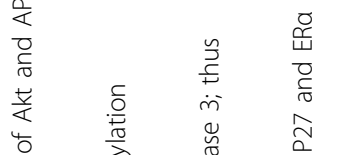

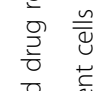

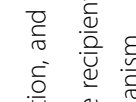

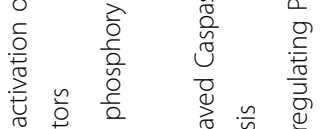

:



c.

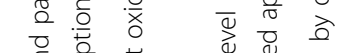

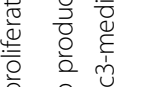

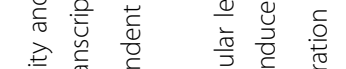

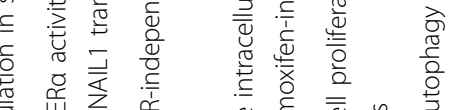

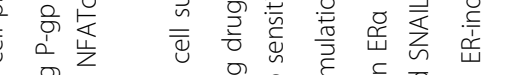

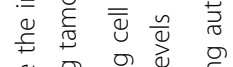

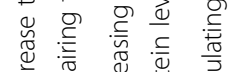

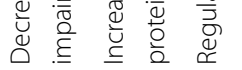

\section{它}

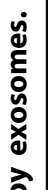

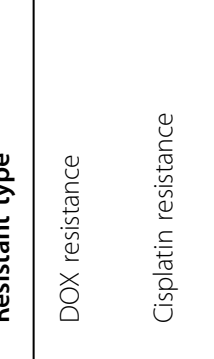
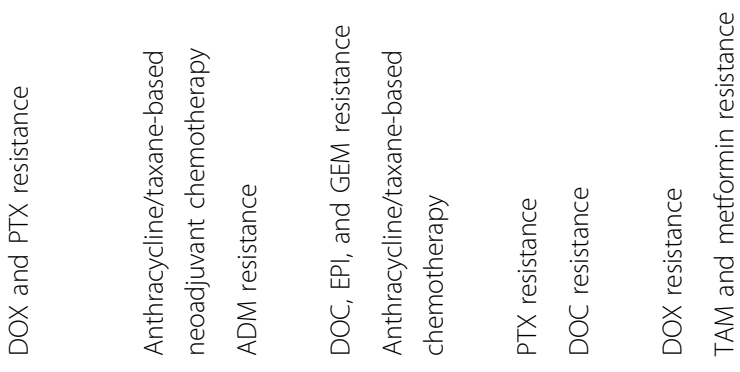

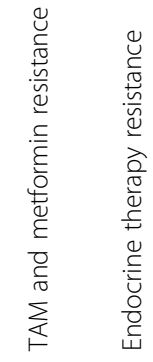

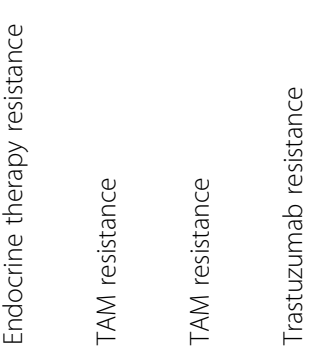

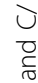



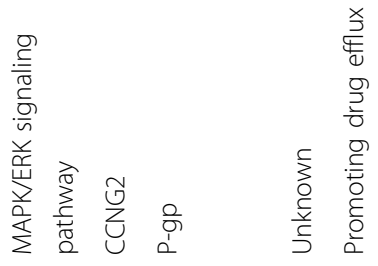

政



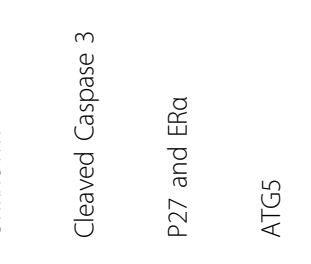

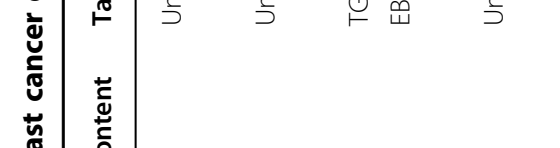



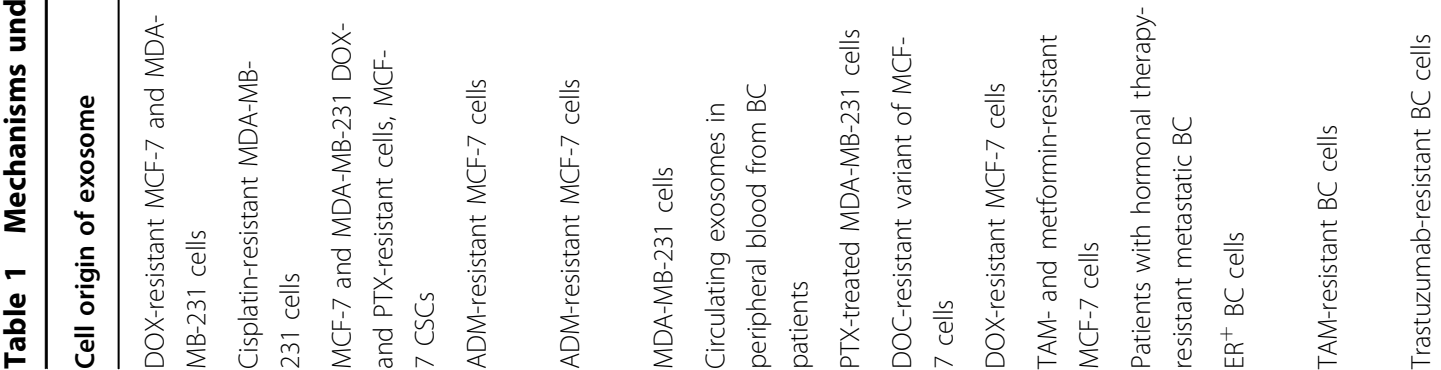




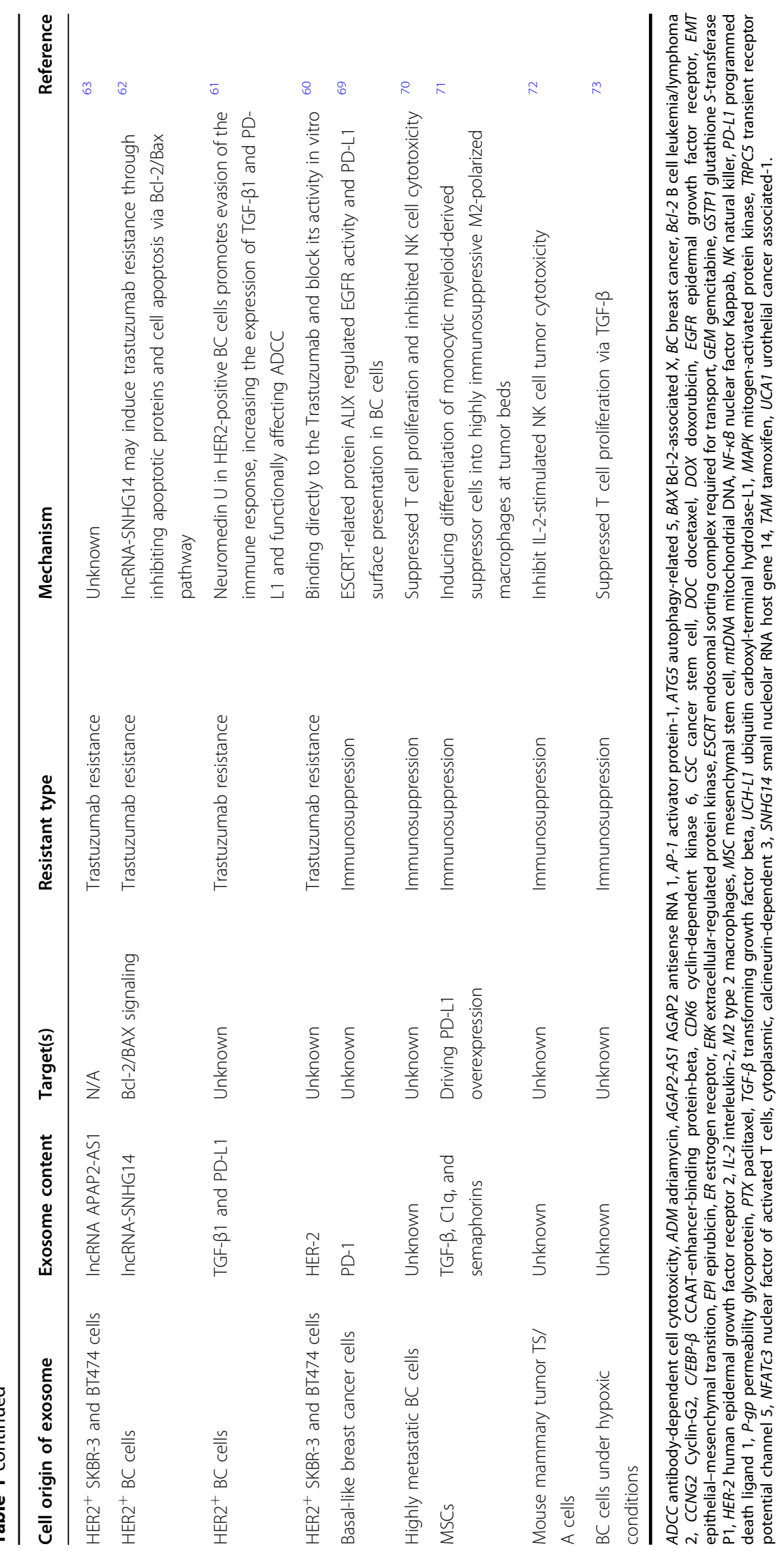




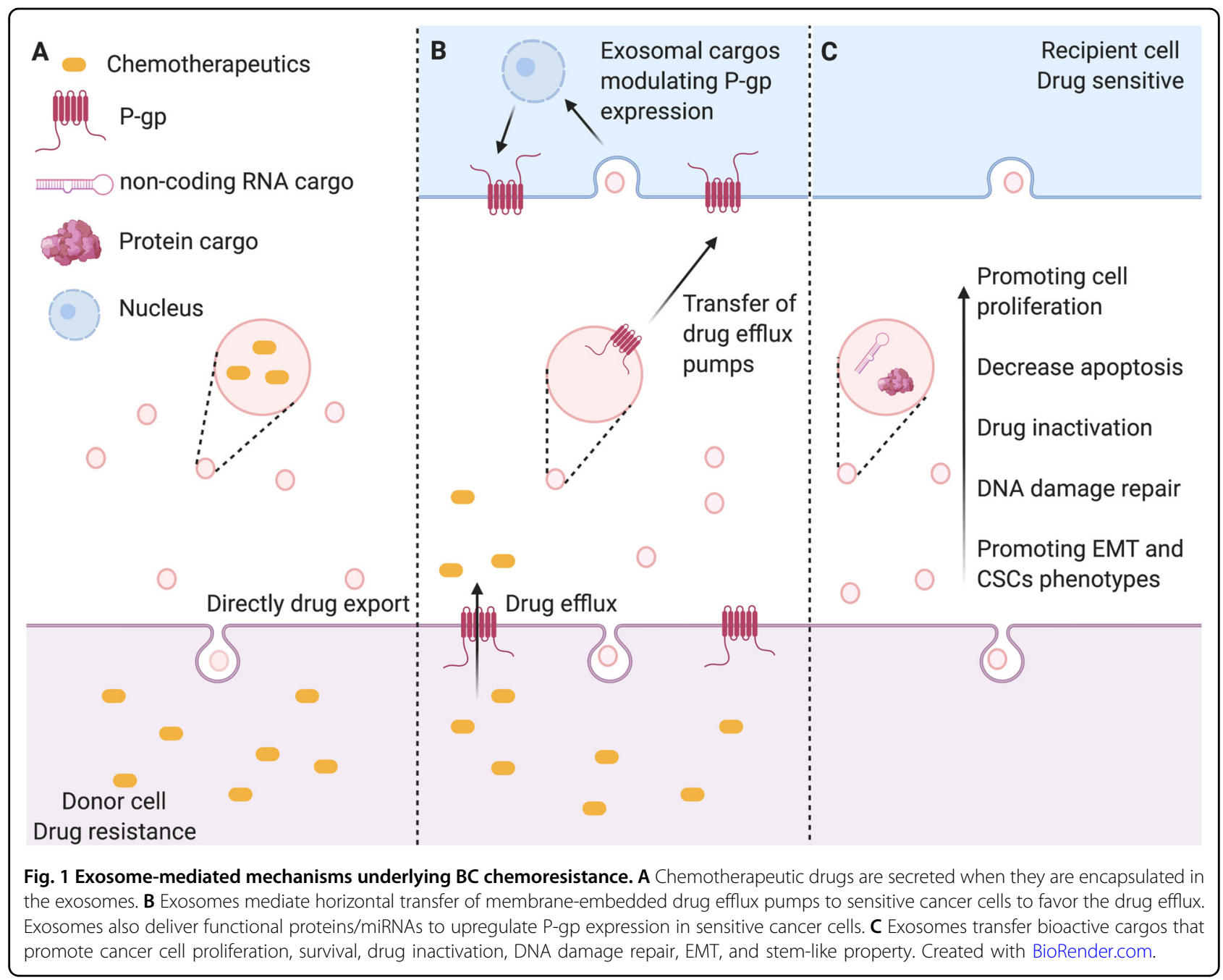

resistance. The third mechanism is the exosome-mediated transfer of functional proteins/miRNAs to modulate P-gp expression (Fig. 1b). Transient receptor potential channels (TRPCs) play a crucial role in the upregulation of P-gp in drug-resistant $\mathrm{BC}$ cells $^{34}$. It is implicated in the adriamycin (ADM) resistance mediated by the exosomes from ADM-resistant MCF-7 cells. The internalization of TRPC5-containing exosomes led to $\mathrm{Ca}^{2+}$ influx through TRPC5 in sensitive MCF-7 cells, which resulted in the upregulation of $\mathrm{P}^{-\mathrm{gp}^{35,36}}$. Furthermore, exosomal ubiquitin carboxyl-terminal hydrolase-L1 (UCH-L1) was also found to upregulate P-gp expression by activating the mitogen-activated protein kinase/extracellular-regulated protein kinase signaling pathway. ADM-resistant MCF-7derived exosomes were enriched with both P-gp and UCH-L1 proteins. Treatment of sensitive MCF-7 cells with the UCH-L1-specific inhibitor LDN-57444 prevented drug resistance induced by the internalization of exosomes from ADM-resistant MCF-7 cells ${ }^{37}$. These results suggest that exosomes play an important role in the chemoresistance of $\mathrm{BC}$ by both directly exporting chemotherapeutic drugs and mediating the transfer or regulation of drug efflux pump.

On the other hand, exosomes also transfer drugmetabolizing enzymes to induce drug inactivation (Fig. 1c). Glutathione $S$-transferase P1 (GSTP1), which belongs to the family of phase II drug-metabolizing enzymes, can detoxify a variety of anticancer drugs by conjugating them with glutathione ${ }^{38}$. Yang et al. ${ }^{39}$ found that the expression of GSTP1 was much higher in ADM-resistant cells and their secreted exosomes. Exposure of sensitive cells to these exosomes induced sensitive cells to develop a drugresistant phenotype.

\section{Exosome-mediated transfer of pro-survival cargo}

In addition to disrupting the intracellular accumulation of drugs, exosomes also transfer functional cargos that activate pro-survival signaling and unchecked cell cycle progression, which is favorable for tumor growth and progression (Fig. 1c). For instance, survivin is a pro- 
survival protein that exists in exosomes from different cancer types ${ }^{40,41}$. Kreger et al. ${ }^{42}$ reported that paclitaxel (PTX) treatment induced the secretion of survivinenriched exosomes from MDA-MB-231 cells, which highly promoted the survival of PTX-treated fibroblasts and SK-BR-3 cells. Moreover, different non-coding RNAs are found in exosomes to favor the survival of chemoresistant $\mathrm{BC}$ cells during treatment. Exosomes derived from cisplatin-resistant MDA-MB-231 cells are characterized by high expression of miR-423-5p. They transferred cisplatin-resistant phenotypes to recipient cells by promoting the proliferation, metastasis, and anti-apoptotic signaling $^{26}$. Furthermore, Wang et al. ${ }^{25}$ found that a high level of exosomal long non-coding RNA (lncRNA)-H19 caused DOX resistance in BC cells. Inhibition of lncRNAH19 significantly reduced the resistance to DOX. Similarly, exosomal miR-1246 was found to promote DOC, epirubicin, and gemcitabine resistance by inhibiting Cyclin-G2 in BC cells ${ }^{43}$. Accordingly, these studies suggest that the transfer of functional proteins and noncoding RNA mediated by exosomes is one of the mechanisms of chemoresistance.

To date, several large-scale validation studies were performed to determine the exosomal protein and miRNA expression profiles in $\mathrm{BC}$ after chemotherapy ${ }^{44-48}$. These studies would provide a comprehensive understanding of the function of dysregulated exosomal miRNAs and proteins, which might be helpful to understand the transmission of chemoresistance mediated by exosomes and to overcome chemoresistance in $\mathrm{BC}$ treatment. Besides, differentially expressed non-coding RNAs and proteins in exosomes from chemoresistant BC cells support their potential use as disease biomarkers to predict chemotherapeutic response in $\mathrm{BC}$ patients.

\section{Exosome and hormonal resistance in $\mathrm{BC}$}

Since approximately $70 \%$ of $\mathrm{BC}$ patients carry tumors with a high level of estrogen receptor- $\alpha(E R \alpha)$, targeting $E R \alpha$ is an effective hormone therapy ${ }^{49,50}$. A variety of ERblocking drugs are now used to treat $\mathrm{ER}^{+} \mathrm{BC}$ patients. Tamoxifen (TAM) is such a drug that can competitively block the ER activation and effectively inhibit $\mathrm{ER}^{+}$tumor growth ${ }^{51}$. However, the effectiveness of hormone therapy in $\mathrm{BC}$ is often terminated by the progression of tumors that develop an acquired hormonal resistance after longterm treatment ${ }^{52,53}$.

The mechanism of hormonal resistance is well studied. The $E R \alpha$ dysregulation, the imbalance between receptor activators and inhibitors, and the activation of hormone-independent pathways are the major causes of hormonal resistance ${ }^{50,53-55}$. Semina et al. $^{56}$ demonstrated that exosomes from TAMresistant MCF-7 cells induced horizontal hormone resistance in estrogen-dependent MCF-7 cells and revealed some key proteins involved. They also found that the co-culture of sensitive MCF-7 cells with exosomes from resistant cells for 14 days caused sensitive cells to develop resistance to antiestrogen drugs ${ }^{54}$.

Exosomal non-coding RNAs play a critical role in the hormonal resistance of BC. Exosomal transfer of lncRNA urothelial cancer associated-1 (UCA1) promoted TAM resistance in $\mathrm{ER}^{+}$MCF-7 cells through mammalian target of rapamycin signaling pathway ${ }^{57}$. Furthermore, exosomal miR-222 mediated TAM resistance in $\mathrm{ER}^{+} \mathrm{BC}$ cells. The expression of miR-222 is upregulated in exosomes secreted from MCF-7 TAM-resistant cells. Given that the downregulation of miR-222 increased the expression of p27 and ER $\alpha$ at both the mRNA and protein levels and restored the sensitivity to TAM, exosomal miR-222 may promote TAM resistance by upregulating $\mathrm{p} 27$ and ER $\alpha$ in $\mathrm{ER}^{+} \mathrm{BC}$ cells ${ }^{58}$. In addition, Sansone et al. ${ }^{59}$ demonstrated that the horizontal transfer of mitochondrial DNA via exosomes promoted treatment-induced metastasis of cancer stem-like cells from dormancy and led to hormonal resistance in $\mathrm{BC}$.

Overall, the acquired hormone resistance of $\mathrm{BC}$ cells can be transferred by exosomes mainly through the following mechanisms: exosomal miRNA- and proteinmediated ER $\alpha$ dysregulation and activation of hormoneindependent pathways. Since few studies showed that hormonal resistance was transferred between $\mathrm{BC}$ cells, further studies are needed to analyze the exosomes secreted by hormonal resistance $\mathrm{BC}$, including the proteome and non-coding RNA profiles, and to determine the key factors for exosome-mediated transferring of the hormone-resistant phenotype.

\section{Exosome and resistance to human epidermal growth factor receptor 2 (HER2)-targeted therapy in $B C$}

Overexpression of HER2 is associated with poor prognosis of $\mathrm{BC}^{60}$. Hence, HER2-targeted therapy has achieved excellent efficacy in the treatment of HER2 ${ }^{+} \mathrm{BC}^{61}$. Trastuzumab is the first monoclonal HER-2 antibody approved for the treatment of HER2 ${ }^{+} \mathrm{BC}$, which significantly improves long-term disease-free survival of $\mathrm{BC}$ patients $^{62}$. Although these drugs have a good clinical response in $\mathrm{BC}$ patients initially, a majority of patients turn refractory to HER2-targeted drugs as early as 1 year after treatment completion ${ }^{63}$.

Some studies have shown that exosome-mediated neutralization of antibody-based drugs is involved in $\mathrm{BC}$ trastuzumab resistance (Fig. 2). Exosomes isolated from SK-BR-3 and BT-474 BC cell lines overexpress HER2, which can directly bind to the trastuzumab and block its activity in vitro ${ }^{64}$. Similar results were also observed in patients' samples.

Martinez et al. ${ }^{65}$ showed that resistance to HER2targeted drugs was related to the increased levels of 


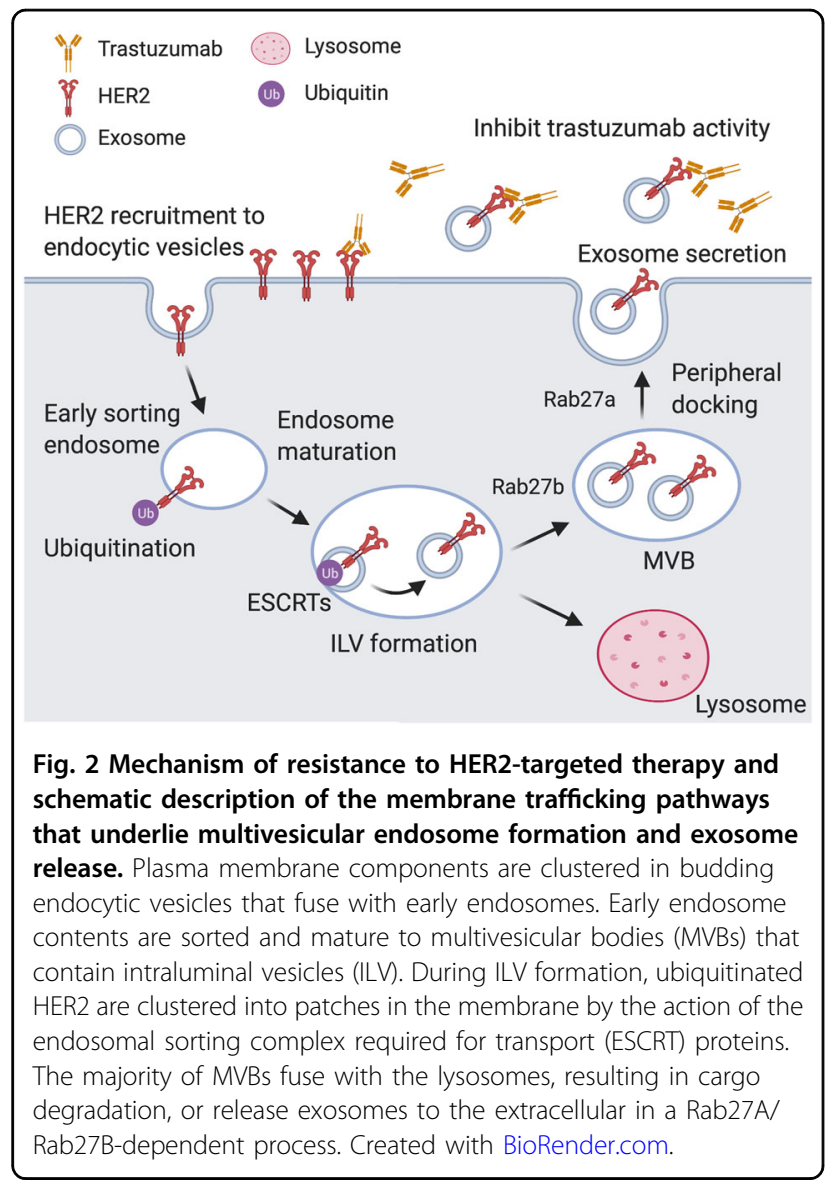

transforming growth factor beta 1 (TGF- $\beta 1$ ) and programmed death-ligand 1 (PD-L1). They found that EVs transfer these molecules to induce the characteristics of their source cells in drug-sensitive cells. EV-associated TGF- $\beta 1$ levels are related to the response to HER2targeted treatment in $\mathrm{HER}_{2}{ }^{+} \mathrm{BC}$ patients, suggesting that it could be used as a biomarker of therapeutic response.

Dysregulation of non-coding RNAs may also contribute to the trastuzumab resistance. The trastuzumab-resistant BC cells secreted exosomes loaded with the lncRNA-small nucleolar RNA host gene 14 (SNHG14) that induced trastuzumab resistance by inhibiting cell apoptosis via $\mathrm{B}$ cell leukemia/lymphoma 2 (BCL-2)/Bcl-2-associated $\mathrm{X}$ pathway. Also, the exosomal expression of lncRNASNHG14 in serum was highly upregulated in patients who exhibited resistance to trastuzumab compared with patients who responded to trastuzumab ${ }^{66}$. Similarly, the lncRNA AGAP2 antisense RNA 1 (AGAP2-AS1)-containing exosomes also enhanced trastuzumab resistance in $\mathrm{BC}$ cells ${ }^{67}$. The heterogeneous nuclear ribonucleoprotein A2B1 (hnRNPA2B1), an RNA-binding protein, was found essential in loading lncRNA AGAP2-AS1 into exosomes $^{68}$. However, the exact mechanism by which exosomal AGAP2-AS1 contributes to trastuzumab resistance needs further investigation. Additionally, by analyzing publicly available miRNA expression profiling data of BC in the Gene Expression Omnibus database, Han et al. ${ }^{69}$ found that the expression of miR-567 was downregulated during trastuzumab resistance. Interestingly, they also found that exosomal miR-567 reversed trastuzumab resistance via inhibiting autophagy-related 5 , whereas knockdown of miR-567 induced resistance to trastuzumab treatment.

Accordingly, these findings reveal the unique role of exosomes in promoting resistance to targeted therapies, which results from the direct interaction of HER2overexpressing exosomes with targeted drugs or from the exosome-mediated transcriptional alternation that promotes cell survival by HER2-independent pathway.

\section{Exosome and immunotherapeutic resistance in BC}

The recent success of novel anticancer immunotherapies has led to a new era of cancer treatment. BCs were traditionally thought to be poorly immunogenic compared to other cancers, such as bladder cancer, melanoma, and lung cancer ${ }^{70}$. However, recent studies demonstrated that the tumors of TNBC are more immunogenic (showing a higher degree of lymphocyteinfiltrating and higher PD-L1 expression) than the other $\mathrm{BC}$ subtypes ${ }^{71}$. Currently, several clinical trials are investigating immune checkpoint programmed death 1/PD-L1 inhibitors as monotherapy or in combination with other therapies, such as chemotherapy, radiotherapy, poly adenosine ribose polymerase inhibitors, or angiogenesis inhibitors in patients with metastatic $\mathrm{TNBC}^{72}$. Based on the results of the IMpassion 130 study, anti-PD-L1 drug atezolizumab plus nab-paclitaxel received accelerated approval on March 8, 2019 from the Food and Drug Administration for unresectable locally advanced or metastatic TNBC with PD-L1 expression ${ }^{3}$. However, there is still much work needed to optimize immunotherapy strategies in $\mathrm{BC}$, and identification and implementation of novel biomarkers for predicting immunotherapeutic response are urgently required.

Recent evidence suggests that exosomes also play essential roles in remodeling the tumor immune microenvironment. It has been reported that PD-L1 could be packaged into the exosomes of tumor cells, and exosomal PD-L1 enables cancer cells to evade antitumor immunity by inhibiting $\mathrm{T}$ cell activation ${ }^{73}$. Also, exosomal PD-L1 appears to be resistant to anti-PD-L1 antibody blockade ${ }^{74}$. However, PD-L1 expression is heterogeneous and dynamic among different BCs. Among different $\mathrm{BC}$ subtypes, it has been proved that basal-like $\mathrm{BC}$ cells constitutively express the highest levels of $\mathrm{PD}-\mathrm{L} 1^{75}$. Monypenny et $\mathrm{al}^{76}$ showed that the endosomal sorting complex required for transport-related protein ALIX regulated epidermal growth factor receptor activity and 
PD-L1 surface presentation in BC cells. They found that ALIX-depleted cells exhibited increased surface levels of PD-L1, conferring an enhanced immunosuppressive phenotype on BC cells. Furthermore, Wen et al. ${ }^{77}$ showed that exosomes derived from highly metastatic $\mathrm{BC}$ cells directly suppressed $\mathrm{T}$ cell proliferation and inhibited natural killer (NK) activity and hence likely suppressed the anticancer immune response in pre-metastatic organs. Zhang et al. ${ }^{78}$ reported that the murine mammary tumor TS/A cell line-derived exosomes inhibited interleukin-2 (IL-2)-stimulated NK cell activity, while the polyphenol derived from the diet, curcumin, reversed the immunosuppressive effects of exosomes on NK cells. In addition, another study demonstrated that exosomes derived from BC cells suppressed T cell proliferation via TGF- $\beta^{79}$. On the other hand, tumor-associated macrophages are major contributors to malignant progression and resistance to immunotherapy. Biswas et al. ${ }^{80}$ reported that mesenchymal stem cell (MSC)-derived exosomes promoted BC progression by inducing differentiation of monocytic myeloid-derived suppressor cells into highly immunosuppressive type 2 macrophage-polarized macrophages at tumor beds. The findings have significant implications for understanding the underlying mechanisms of immunosuppression in the TME of BC.

In summary, exosomes carry immunosuppressive molecules known to affect immune cell functions in several different ways, which is well studied in different tumors. As most of the immunotherapy research of $\mathrm{BC}$ is in the clinical trial stage, the study of exosome-mediated immunosuppression is still at the beginning and needs to be further studied. Exosomes may be used as a predictive biomarker that could potentially serve as a non-invasive strategy to monitor immunotherapy response in tumors.

\section{Exosomes regulate EMT, cancer stem cell (CSC), and TME in the drug resistance of $B C$} Exosome-mediated enhanced EMT and stemness in the drug resistance of $B C$

Recent studies have shown that the release characteristics of EVs are usually coupled with cellular phenotypic transformation, including $\mathrm{EMT}^{81,82}$ and $\mathrm{CSC}^{83,84}$. EMT and stemness promote the EV-releasing phenotype of cells, and tumor-derived EVs could in turn initiate EMT and stemness in tumor cells ${ }^{85}$. Thus it is conceivable that the EMT and CSC phenotypes are involved in promoting tumor progression and the acquisition of therapeutic resistance mediated by exosomes.

EMT is a biologic process of loss of epithelial characteristics and the acquisition of mesenchymal phenotype $^{86}$. Cells with EMT undergo a variety of biochemical changes, such as the loss of the tight cell-cell adhesion and the gaining of invasive, migrative, and anti-apoptotic ability. The exosome is an important mediator of EMT that transforms cancer cells into more aggressive phenotypes. Increasing studies indicated that exosomes could deliver pro-EMT factors to recipient cells, thereby facilitating $\mathrm{BC}$ progression, including anti-apoptosis, invasion, metastasis, and chemoresistance ${ }^{87-90}$. The miR-155 is an important regulator in EMT and $\mathrm{CSCs}^{91}$. Santos et al. ${ }^{90}$ reported that miR-155 upregulation in DOX- and PTXresistant cells was associated with EMT. The co-culture of DOX- and PTX-sensitive cells with exosomes derived from CSCs or DOX- and PTX-resistant cells resulted in increased levels of miR-155 and induction of chemoresistance. In addition to these effects, EMT is also induced in cells when transfected with miR-155. Qin et al. ${ }^{89}$ reported that TGF- $\beta 2$ was significantly upregulated in breast milk exosomes during weaning/early involution. Exosomes loaded with a high level of TGF- $\beta 2$ can lead to EMT in both cancer and benign cells, with the change of cell morphology and actin cytoskeleton, loss of cell-cell junction, increased alpha-smooth muscle actin and vimentin, and decreased E-cadherin.

CSCs are characterized by self-renewal ability and can differentiate into multiple subpopulations of cells within tumors. CSCs not only drive tumor initiation and growth but also mediate tumor metastasis and therapeutic resistance ${ }^{92}$. Shen et al. ${ }^{93}$ showed that treatment with a sublethal dose of chemotherapeutics induced $\mathrm{BC}$ cells to secrete EVs, which stimulated a CSC phenotype, and made cancer cells resistant to therapy. Chemotherapy induced $\mathrm{BC}$ cells to secrete multiple EV miRNAs, including miR-9-5p, miR-195-5p, and miR-203a-3p, which simultaneously targeted the transcriptional factor one cut homeobox 2 (ONECUT2), leading to the induction of CSC phenotypes and expression of stemnessassociated genes, including NOTCH1, SOX9, NANOG, OCT4, and SOX2. Inhibition of these miRNAs or restoration of ONECUT2 expression abolished the CSCstimulating effect of EVs from chemotherapy-treated cancer cells.

These findings reveal a critical mechanism of resistance to chemotherapy by which $\mathrm{BC}$ cells secrete miRNAcontaining EVs to stimulate EMT- and CSC-like features.

\section{Directional transfer of drug resistance via exosomes between TME and BC cells}

The TME is a heterogeneous population of cells that can be broadly organized into endothelial cells, cancerassociated fibroblasts, and immune cells ${ }^{94}$. Moreover, there are also some non-cellular components, including the extracellular matrix and various soluble cytokines, chemokines, and $\mathrm{EVs}^{95}$. It is becoming well established that there are substantial crosstalks between surrounding cells and cancer cells within the TME, promoting tumor development, progression, and drug resistance ${ }^{96}$. TME is characterized with hypoxia, acidic, high oxidative stress ${ }^{97}$. 


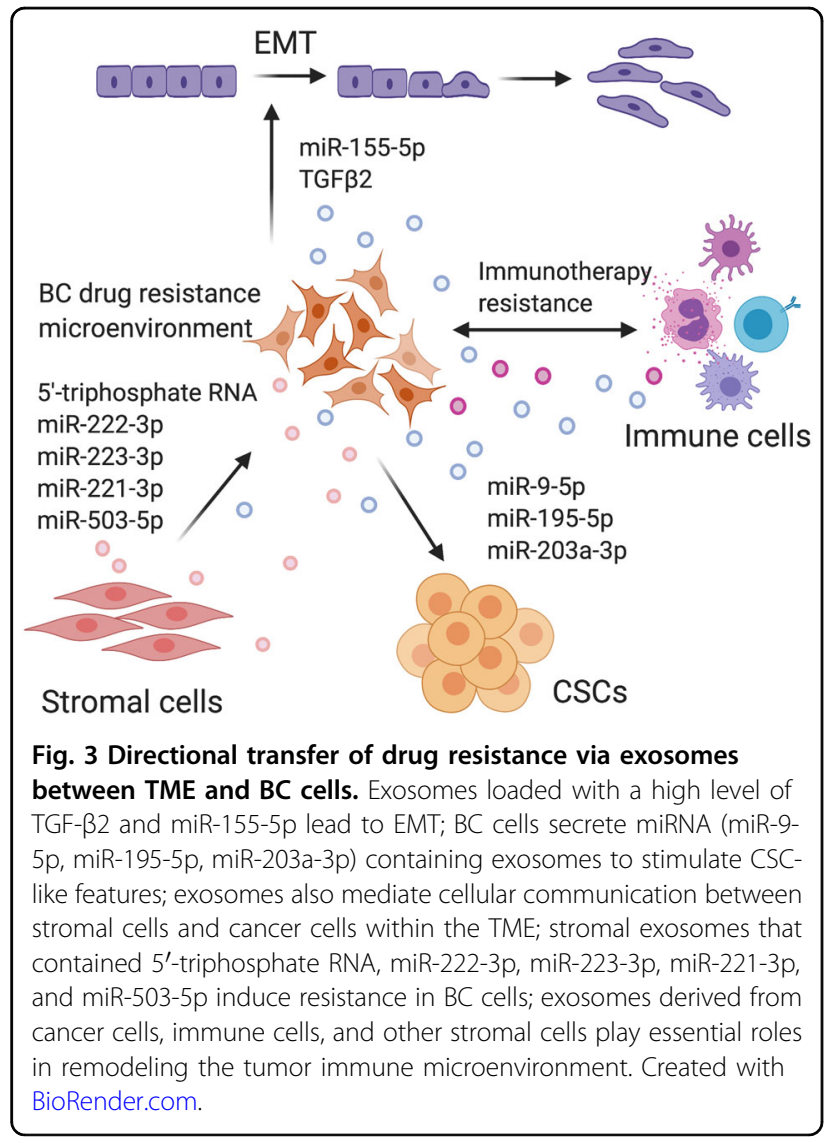

Recent emerging evidence suggests that all these characteristics of TME promote exosome secretion and regulate the content of exosomes by cancer cells, thereby increasing intercellular communication at local and distance sites ${ }^{98-101}$. Here we summarize that, in BC cells, exosomes, as carriers of information, mediate communications between the various cell types in TME leading to the acquisition of drug resistance (Fig. 3).

A variety of examples of exosome transfer between cancer cells and stromal cells that lead to drug resistance have now been reported. Stromal exosomes that contained $5^{\prime}$-triphosphate RNA induced resistance in BC cells through the viral RNA pattern recognition receptor retinoic acid-inducible gene I (RIGI) and NOTCH3 signaling and activated a signal transducer and activator of transcription 1 (STAT1)-dependent antiviral response ${ }^{102}$. STAT1 is one target of interferon-related DNA damage resistance signature, and it was shown that stromal cells increased radioresistance and chemoresistance by communicating with $\mathrm{BC}$ cells in a STAT1-dependent manner ${ }^{102}$. The RAB27B, located at chromosome 18q21.1, is a member of the Rab subfamily of small GTPases ${ }^{103}$. RAB27B promoted the transfer of exosomes secreted from stromal cells to BC cells, then exosomal 5'-triphosphate RNA increased the expression of RIGI, which enhanced $\mathrm{BC}$ radioresistance by inducing interferonrelated DNA damage resistance signature expression ${ }^{102}$. The same group reported another study that exosomes derived from stromal fibroblasts contain unshielded RN7SL1 RNA that could also be transferred to immune cells, and the unshielded RN7SL1 drives an inflammatory response by increasing the percentage of myeloid dendritic cell (DC) populations ${ }^{104}$.

In addition, the crosstalk between tumors and the TME also regulates tumor progression and response to anticancer drugs by exosomal miRNAs. MSCs release miR$222 / 223$-containing exosomes that induce quiescence in a proportion of $\mathrm{BC}$ cells and induce drug resistance ${ }^{105}$. Increased exosomal miR-503 in plasma was detected in BC patients treated with neoadjuvant chemotherapy, which could be partly due to increased miRNA secretion by endothelial cells. The modulation of miR-503 in BC cells altered their proliferative and invasive capacities. This reveals the involvement of the endothelium in the modulation of tumor progression via the secretion of circulating miR-503 in response to chemotherapy ${ }^{106}$.

TME also contains infiltrated immune cells, such as macrophages, neutrophils, mast cells, myeloid-derived suppressor cells, DCs, and NK cells, and lymphocytes ${ }^{107}$. These cells secrete exosomes to transmit the information of immune activation or immunosuppression, and exosomes carrying tumor-associated antigens interfere with antitumor immunotherapies, which have been discussed in the "Exosome and immunotherapeutic resistance in $\mathrm{BC}$ " section.

In summary, at present, the research on exosomes and TME in BC is still limited, and a large number of profiling studies are needed. The evidence we discuss above suggests that exosomes are released by cells within TME in response to antitumor therapies and mediate crosstalk in the TME for the transfer of drug resistance to BC cells. All of these studies indicate that exosomes have a potential role as tumor biomarkers to reflect TME changes and predict therapeutic response.

\section{Potential clinical applications of exosomes in BC drug resistance}

Exosomes as biomarkers on the prediction of therapeutic response in $\mathrm{BC}$

Biomarkers can be used to predict the response of a tumor to a specific treatment and may reflect tumor sensitivity or drug resistance. Although there has been extensive research on biomarkers in BC for prediction of therapeutic response, few are currently recommended for routine clinical applications ${ }^{108}$. Recently, blood-based monitoring of treatment response and progression via liquid biopsy analysis (circulating tumor cells, cell-free DNA, exosomes, and proteins) is attractive because it is minimally invasive, repeatable, and allows a dynamic assessment of specific molecular markers ${ }^{109,110}$. 
With the understanding of exosome biology and its relationship with tumor therapy resistance, the unique features of exosomes (carrying surface markers, their cargos reflect the physiological state of the cell they originated from, relatively stable structure, and presence in almost all biological fluids) make them attractive for the study of early detection of disease progression and as potential biomarkers of response or resistance to therapy ${ }^{111-113}$. Indeed, exosomes have some advantages over other analyses used in liquid biopsies. Exosomes contain molecules from their parental cells and protect their cargos from degradation. Therefore, the analysis of nucleic acid contained in exosomes may be more informative and reproducible than that of vesicle-free circulating tumor RNA or circulating tumor DNA (ctDNA). Second, exosomes are more abundant than cell-free DNA, and they provide the opportunity to get information at the DNA, RNA, and protein level. Combination of the information obtained from exosomes and ctDNA may give more accurate results or information for prediction of therapeutic response in $\mathrm{BC}$ and monitoring $\mathrm{BC}$ progression.

Several recent studies have investigated exosome-associated biomarkers in patients undergoing chemotherapy. Wang et $\mathrm{all}^{35}$ found that the circulating exosomes carrying TRPC5 were significantly correlated with the expression level of TRPC5 in BC tissues and response to chemotherapy. In addition, increased circulating exosomes carrying TRPC5 after chemotherapy preceded cancer progression and predicted acquired chemotherapy resistance. Therefore, the detection of TRPC5-positive exosomes can be used to monitor chemotherapy resistance in real time. Another group found that the level of serum exosomal lncRNA HOTAIR from BC patients was significantly higher than that in healthy individuals ${ }^{114}$. Importantly, the level of exosomal lncRNA HOTAIR significantly decreased in all patients 3 months after the operation, suggesting that the source of serum HOTAIR is from the tumor tissue, and its level is related to tumor burden and disease invasiveness. Also, a high expression level of serum exosomal HOTAIR was associated with a poorer response to neoadjuvant chemotherapy and TAM therapy ${ }^{114}$. Stevic et al. ${ }^{115}$ showed that some miRNAs were selectively enriched in exosomes of HER2 ${ }^{+} \mathrm{BC}$ and TNBC and were also related with the clinicopathological parameters and pathological complete response within the $\mathrm{BC}$ subtypes. The levels of exosome-associated miR-155 and miR-301 changed significantly after neoadjuvant therapy.

Large-scale validation studies on exosome biomarkers can offer significant insight into tumor therapeutic monitoring. However, there are still some limitations. For instance, a standardized method for collecting, processing, and separation of the exosome sample has not been established. Current separation techniques, such as ultracentrifugation, are time-consuming and cannot achieve high-purity separation. Some alternative methods have been developed based on separation by size, immunoaffinity trapping, and exosome precipitation. However, these methods fail to isolate exosomes exclusively and usually result in complex mixtures of EVs and other components of the extracellular space due to overlapping characteristics ${ }^{116}$. In addition, some microfluidic technologies, such as nano-plasmonic exosome technology, in which the label-free exosome detection is based on the surface plasmon resonance, is not widely used $^{117}$. The process of exosome enrichment is still being optimized, and each process may be optimized for a specific cargo, such as protein, DNA, and RNA ${ }^{118,119}$. Also, the application of exosomes as a biomarker is a challenge, because in circulation tumor-derived exosomes are mixed with exosomes derived from normal cells, which might affect the ability to identify and analyze tumor-derived exosomes. To overcome this, we need to identify tumor-specific exosomes for cancer diagnosis or monitoring. Our laboratory has recently developed a multiplexed assay to detect tumor-specific exosomes for cancer early diagnosis using a panel of existing cancer antibodies (unpublished data). Recently, Hoshino et al. ${ }^{120}$ analyzed 426 human samples by proteomic profiling to identify and characterize tumor-derived EV markers in human tissues and plasma different from normal controls, which could be helpful for tumor detection, determining cancer type, and even tumor therapeutic monitoring.

\section{Exosomes as novel therapeutic interventions in $\mathrm{BC}$ drug resistance}

As discussed above, exosomes serve as mediators to promote drug resistance by transferring specific proteins or RNAs. There are several strategies to mitigate the role of exosomes in transferring drug resistance in $\mathrm{BC}$, including blocking exosome secretion from specific cell types, such as stromal and $\mathrm{BC}$ cells ${ }^{121,122}$, and inhibiting the incorporation of drug transporter into exosomes that can lead to redistribution and accumulation of drugs in BC cells ${ }^{123,124}$. Furthermore, stimulating the immune response of a cancer patient by removing immunosuppressive exosomes with specific biomarkers from circulation using an extracorporeal filter is another strategy ${ }^{125}$. However, there are still many problems in these therapeutic strategies, such as the lack of specific biomarkers and inhibitors.

Several studies have shown the advantages of the utility of exosomes as naturally derived delivery vehicles for therapeutic agents and vaccines for tumor immune therapy. These advantages include nanoscale size, immune compatibility, low toxicity, prolonged blood circulation time, and relatively stable ${ }^{126}$. As natural carriers, exosomes are safe and effective for targeted drug delivery or therapy. Recently, Li et al. ${ }^{127}$ developed a macrophage-derived exosome-coated poly (lactic-co-glycolic acid) nanoplatform for targeted chemotherapy of TNBC. Similarly, Naseri et al. ${ }^{128}$ exploited the exosomes isolated from bone marrow-derived MSCs to 
deliver LNA (locked nucleic acid)-modified anti-miR-142-3p oligonucleotides to suppress the expression level of miR-142$3 \mathrm{p}$ and miR-150 in 4T1 and TUBO BC cell lines. Aqil et al. ${ }^{129}$ showed that curcumin can be delivered effectively using milk-derived exosomes. Oral administration of exosomal curcumin demonstrated enhanced antiproliferative, antiinflammatory, and antitumor activities against multiple cancer cell lines including BC compared with the free curcumin. In addition, many peptides, non-coding RNAs, or chemotherapeutic agents can be loaded into exosomes derived from distinct cells using different loading methods and targeted strategies, which makes exosomes as an efficient carrier to enhance antitumor therapy and reverse drug resistance.

It has been shown that tumor-derived exosomes contain and transfer tumor-associated antigens, as well as major histocompatibility complex class I molecules, to DC and consequently induce a $\mathrm{T}$ cell-mediated immune response against tumor cells ${ }^{130}$. In addition, DC-derived exosomes have been proven safe for vaccine delivery in multiple phase I trials in different types of cancers ${ }^{131}$. Li et al. ${ }^{132}$ developed a novel HER2-specific exosome-T vaccine using polyclonal $\mathrm{CD} 4^{+} \mathrm{T}$ cells armed with HER2-specific exosomes released from $\mathrm{DC}$ and demonstrated its therapeutic effect against $\mathrm{HER}^{+} \mathrm{BC}$ in double-transgenic HER2/HLA-A2 mice. This study provides a new therapeutic alternative for trastuzumab-resistant BC patients with HER2-specific self-immune tolerance.

\section{Conclusions}

Although modern antitumor drugs have made significant progress, the occurrence of drug resistance often leads to treatment failure in $\mathrm{BC}$. The exosome is a kind of EVs found in circulation, which has the unique potential to capture the dynamic complexity of cancer and can be used to measure a variety of biological components associated with tumor drug resistance in real time. Despite numerous challenges, exosomes may be used as a candidate biomarker for predicting and monitoring therapeutic efficacy in $\mathrm{BC}$ patients and as a potential target or carrier to reverse drug resistance, which occupies an important position in the future of tumor detection, prediction, and treatment.

\section{Acknowledgements}

This paper was partly supported by the National Natural Science Foundation of China (81802924) and the St George Cancer Care Centre Research Trust Fund. The authors would also like to thank the funding support from the UNSW Sydney and the China Scholarship Council.

\footnotetext{
Author details

'Department of Biopharmaceutical Sciences, College of Pharmacy, Harbin Medical University, 150081 Harbin, Heilongjiang, China. ${ }^{2}$ St George and Sutherland Clinical School, Faculty of Medicine, UNSW Sydney, Kensington, NSW 2052, Australia. ${ }^{3}$ Cancer Care Centre, St. George Hospital, Kogarah, NSW 2217, Australia. ${ }^{4}$ Institute of Precision Cancer Medicine and Pathology and Department of Pathology, Jinan University Medical College, 510630 Guangzhou, China. ${ }^{5} \mathrm{School}$ of Medicine and Centre for Molecular and Medical
}

Research, Deakin University, Waurn Ponds, VIC 3216, Australia. ${ }^{6}$ School of Basic Medicine Sciences, Zhengzhou University, 450001 Henan, China

\section{Author contributions}

X.D. and Y.L. conceived the structure of the manuscript. X.D. wrote the manuscript and drew the figures and tables and was a major contributor in writing. X.B., J.N., H.Z., W.D., P.G., and Y.L. revised the manuscript. All authors read and approved the final manuscript.

\section{Conflict of interest}

The authors declare that they have no conflict of interest.

\section{Publisher's note}

Springer Nature remains neutral with regard to jurisdictional claims in published maps and institutional affiliations.

Received: 8 July 2020 Revised: 21 October 2020 Accepted: 23 October 2020 Published online: 17 November 2020

\section{References}

1. Bray, F. et al. Global cancer statistics 2018: GLOBOCAN estimates of incidence and mortality worldwide for 36 cancers in 185 countries. CA Cancer J. Clin. 68, 394-424 (2018).

2. Jayaraj, R. et al. Clinical theragnostic relationship between drug-resistance specific miRNA expressions, chemotherapeutic resistance, and sensitivity in breast cancer: a systematic review and meta-analysis. Cells 8, 1250 (2019).

3. O'Sullivan, H., Collins, D. \& O'Reilly, S. Atezolizumab and nab-paclitaxel in advanced triple-negative breast cancer. N. Engl. J. Med. 380, 986 (2019).

4. Vasan, N., Baselga, J. \& Hyman, D. M. A view on drug resistance in cancer. Nature 575, 299-309 (2019).

5. Lappano, R., Rigiracciolo, D. C., Belfiore, A., Maggiolini, M. \& De Francesco, E. M. Cancer associated fibroblasts: role in breast cancer and potential as therapeutic targets. Expert Opin. Ther. Targets 24, 559-572 (2020).

6. Matei, I., Kim, H. S. \& Lyden, D. Unshielding exosomal RNA unleashes tumor growth and metastasis. Cell 170, 223-225 (2017).

7. Brown, J. M. \& Giaccia, A. J. The unique physiology of solid tumors: opportunities (and problems) for cancer therapy. Cancer Res. 58, 1408-1416 (1998).

8. Taube, J. M. et al. Implications of the tumor immune microenvironment for staging and therapeutics. Mod. Pathol. 31, 214-234 (2018).

9. Sedgwick, A. E. \& D'Souza-Schorey, C. The biology of extracellular microvesicles. Traffic 19, 319-327 (2018).

10. Hendrix, A. \& Hume, A. N. Exosome signaling in mammary gland development and cancer. Int. J. Dev. Biol. 55, 879-887 (2011).

11. Maacha, S. et al. Extracellular vesicles-mediated intercellular communication: roles in the tumor microenvironment and anti-cancer drug resistance. Mol. Cancer 18, 55 (2019).

12. Mao, X. \& Jin, F. The exosome and breast cancer cell plasticity. Onco Targets Ther. 12, 9817-9825 (2019).

13. Ramirez, M. I. et al. Technical challenges of working with extracellular vesicles. Nanoscale 10, 881-906 (2018).

14. Kalluri, R. \& LeBleu, V. S. The biology, function, and biomedical applications of exosomes. Science 367, eaau6977 (2020).

15. Mathieu, M., Martin-Jaular, L., Lavieu, G. \& Théry, C. Specificities of secretion and uptake of exosomes and other extracellular vesicles for cell-to-cell communication. Nat. Cell Biol. 21, 9-17 (2019).

16. Kalluri, R. The biology and function of exosomes in cancer. J. Clin. Investig. 126, 1208-1215 (2016).

17. Goh, C. Y. et al. Exosomes in triple negative breast cancer: garbage disposals or Trojan horses? Cancer Lett. 473, 90-97 (2020).

18. Giallombardo, M., Taverna, S., Alessandro, R., Hong, D. \& Rolfo, C. Exosomemediated drug resistance in cancer: the near future is here. Ther. Adv. Med. Oncol. 8, 320-322 (2016).

19. Ender, F., von Bubnoff, N. \& Gieseler, F. Extracellular vesicles: subcellular organelles with the potential to spread cancer resistance. Anticancer Res. $\mathbf{3 9}$, 3395-3404 (2019).

20. Dallavalle, $\mathrm{S}$. et al. Improvement of conventional anti-cancer drugs as new tools against multidrug resistant tumors. Drug Resist. Updat. 50, 100682 (2020). 
21. Battista, T. et al. Roles of sorcin in drug resistance in cancer: one protein, many mechanisms, for a novel potential anticancer drug target. Cancers $\mathbf{1 2}$, 887 (2020)

22. Navas, $\mathrm{T}$. et al. Clinical evolution of epithelial-mesenchymal transition in human carcinomas. Cancer Res. 80, 304-318 (2020).

23. Garcia-Mayea, Y., Mir, C., Masson, F., Paciucci, R. \& ME, L. L. Insights into new mechanisms and models of cancer stem cell multidrug resistance. Semin. Cancer Biol. 60, 166-180 (2020).

24. LV, M. M. et al. Exosomes mediate drug resistance transfer in MCF-7 breast cancer cells and a probable mechanism is delivery of P-glycoprotein. Tumour Biol. 35, 10773-10779 (2014).

25. Wang, $X$. et al. Exosome-mediated transfer of long noncoding RNA H19 induces doxorubicin resistance in breast cancer. J. Cell. Physiol. 235, 6896-6904 (2020).

26. Wang, B. et al. Cisplatin-resistant MDA-MB-231 cell-derived exosomes increase the resistance of recipient cells in an exosomal miR-423-5pdependent manner. Curr. Drug Metab. 20, 804-814 (2019).

27. Shedden, K., Xie, X. T., Chandaroy, P., Chang, Y. T. \& Rosania, G. R. Expulsion of small molecules in vesicles shed by cancer cells: association with gene expression and chemosensitivity profiles. Cancer Res. 63, 4331-4337 (2003).

28. Locher, K. P. Mechanistic diversity in ATP-binding cassette (ABC) transporters. Nat. Struct. Mol. Biol. 23, 487-493 (2016).

29. Nedeljković, M. \& Damjanović, A. Mechanisms of chemotherapy resistance in triple-negative breast cancer-how we can rise to the challenge. Cells 8, 957 (2019).

30. Ambudkar, S. V., Kimchi-Sarfaty, C., Sauna, Z. E. \& Gottesman, M. M. P-glycoprotein: from genomics to mechanism. Oncogene 22, 7468-7485 (2003).

31. Bebawy, M. et al. Membrane microparticles mediate transfer of Pglycoprotein to drug sensitive cancer cells. Leukemia 23, 1643-1649 (2009).

32. Levchenko, A. et al. Intercellular transfer of P-glycoprotein mediates acquired multidrug resistance in tumor cells. Proc. Natl Acad. Sci. USA 102, 1933-1938 (2005)

33. Sousa, D., Lima, R. T. \& Vasconcelos, M. H. Intercellular transfer of cancer drug resistance traits by extracellular vesicles. Trends Mol. Med. 21, 595-608 (2015).

34. Ma, X. et al. Transient receptor potential channel TRPC5 is essential for Pglycoprotein induction in drug-resistant cancer cells. Proc. Natl Acad. Sci. USA 109, 16282-16287 (2012)

35. Wang, T. et al. Increasing circulating exosomes-carrying TRPC5 predicts chemoresistance in metastatic breast cancer patients. Cancer Sci. 108 448-454 (2017).

36. $\mathrm{Ma}, \mathrm{X}$. et al. Essential role for TrpC5-containing extracellular vesicles in breast cancer with chemotherapeutic resistance. Proc. Natl Acad. Sci. USA 111 6389-6394 (2014).

37. Ning, $\mathrm{K}$. et al. UCH-L1-containing exosomes mediate chemotherapeutic resistance transfer in breast cancer. J. Surg. Oncol. 115, 932-940 (2017).

38. Coles, B. F. \& Kadlubar, F. F. Detoxification of electrophilic compounds by glutathione S-transferase catalysis: determinants of individual response to chemical carcinogens and chemotherapeutic drugs? Biofactors 17, 115-130 (2003).

39. Yang, S. J. et al. Predictive role of GSTP1-containing exosomes in chemotherapy-resistant breast cancer. Gene 623, 5-14 (2017).

40. Khan, S. et al. Extracellular, cell-permeable survivin inhibits apoptosis while promoting proliferative and metastatic potential. Br. J. Cancer 100, 1073-1086 (2009).

41. Khan, S. et al. Survivin is released from cancer cells via exosomes. Apoptosis 16, 1-12 (2011).

42. Kreger, B. T., Johansen, E. R., Cerione, R. A. \& Antonyak, M. A. The enrichment of survivin in exosomes from breast cancer cells treated with paclitaxel promotes cell survival and chemoresistance. Cancers 8, 111 (2016).

43. Li, X. J., Ren, Z. J., Tang, J. H. \& Yu, Q. Exosomal microRNA MiR-1246 promotes cell proliferation, invasion and drug resistance by targeting CCNG2 in breast cancer. Cell. Physiol. Biochem. 44, 1741-1748 (2017).

44. Kavanagh, E. L. et al. Protein and chemotherapy profiling of extracellular vesicles harvested from therapeutic induced senescent triple negative breast cancer cells. Oncogenesis 6, e388 (2017).

45. Chen, W. X. et al. Bioinformatics analysis of dysregulated microRNAs in exosomes from docetaxel-resistant and parental human breast cancer cells. Cancer Manag. Res. 11, 5425-5435 (2019).

46. Ozawa, P. M. M. et al. Extracellular vesicles from triple-negative breast cancer cells promote proliferation and drug resistance in non-tumorigenic breast cells. Breast Cancer Res. Treat. 172, 713-723 (2018).
47. Chen, W. X. et al. Analysis of miRNA signature differentially expressed in exosomes from adriamycin-resistant and parental human breast cancer cells. Biosci. Rep. 38, BSR20181090 (2018).

48. Zhong, S. et al. MicroRNA expression profiles of drug-resistance breast cancer cells and their exosomes. Oncotarget 7, 19601-19609 (2016).

49. Brufsky, A. M. \& Dickler, M. N. Estrogen receptor-positive breast cancer: exploiting signaling pathways implicated in endocrine resistance. Oncologist 23, 528-539 (2018).

50. Muluhngwi, P. \& Klinge, C. M. Identification of miRNAs as biomarkers for acquired endocrine resistance in breast cancer. Mol. Cell. Endocrinol. 456 76-86 (2017).

51. Davies, C. et al. Relevance of breast cancer hormone receptors and other factors to the efficacy of adjuvant tamoxifen: patient-level meta-analysis of randomised trials. Lancet 378, 771-784 (2011).

52. Rani, A., Stebbing, J., Giamas, G. \& Murphy, J. Endocrine resistance in hormone receptor positive breast cancer-from mechanism to therapy. Front. Endocrinol. 10, 245 (2019).

53. Osborne, C. K. \& Schiff, R. Mechanisms of endocrine resistance in breast cancer. Annu. Rev. Med. 62, 233-247 (2011).

54. Semina, S. E. et al. Exosome-mediated transfer of cancer cell resistance to antiestrogen drugs. Molecules 23, 829 (2018).

55. Scherbakov, A. M., Andreeva, O. E., Shatskaya, V. A. \& Krasil'nikov, M. A. The relationships between snail1 and estrogen receptor signaling in breast cancer cells. J. Cell. Biochem. 113, 2147-2155 (2012).

56. Semina, S. E., Scherbakov, A. M., Kovalev, S. V., Shevchenko, V. E. \& Krasil'nikov, M. A. Horizontal transfer of tamoxifen resistance in MCF-7 cell derivates: proteome study. Cancer Investig. 35, 506-518 (2017).

57. Xu, C. G., Yang, M. F., Ren, Y. Q., Wu, C. H. \& Wang, L. Q. Exosomes mediated transfer of IncRNA UCA1 results in increased tamoxifen resistance in breast cancer cells. Eur. Rev. Med. Pharmacol. Sci. 20, 4362-4368 (2016).

58. Wei, Y. et al. Exosomal miR-221/222 enhances tamoxifen resistance in recipient ER-positive breast cancer cells. Breast Cancer Res. Treat. 147, 423-431 (2014).

59. Sansone, P. et al. Packaging and transfer of mitochondrial DNA via exosomes regulate escape from dormancy in hormonal therapy-resistant breast cancer. Proc. Natl Acad. Sci. USA 114, E9066-E9075 (2017).

60. Cortesi, L. et al. Twenty-years experience with de novo metastatic breast cancer. Int. J. Cancer 137, 1417-1426 (2015).

61. Tagliabue, E., Balsari, A., Campiglio, M. \& Pupa, S. M. HER2 as a target for breast cancer therapy. Expert Opin. Biol. Ther. 10, 711-724 (2010).

62. Cameron, D. et al. 11 years' follow-up of trastuzumab after adjuvant chemotherapy in HER2-positive early breast cancer: final analysis of the HERceptin Adjuvant (HERA) trial. Lancet 389, 1195-1205 (2017).

63. Ahmad, A. Current updates on trastuzumab resistance in HER2 overexpressing breast cancers. Adv. Exp. Med. Biol. 1152, 217-228 (2019).

64. Ciravolo, V. et al. Potential role of HER2-overexpressing exosomes in countering trastuzumab-based therapy. J. Cell. Physiol. 227, 658-667 (2012).

65. Martinez, V. G. et al. Resistance to HER2-targeted anti-cancer drugs is associated with immune evasion in cancer cells and their derived extracellular vesicles. Oncoimmunology 6, e1362530 (2017).

66. Dong, $\mathrm{H}$. et al. Exosome-mediated transfer of IncRNASNHG14 promotes trastuzumab chemoresistance in breast cancer. Int. J. Oncol. 53, 1013-1026 (2018).

67. Zheng, Z., Chen, M., Xing, P., Yan, X. \& Xie, B. Increased expression of exosomal AGAP2-AS1 (AGAP2 antisense RNA 1) in breast cancer cells inhibits trastuzumab-induced cell cytotoxicity. Med. Sci. Monit. 25, 2211-2220 (2019).

68. Alarcón, C. R. et al. HNRNPA2B1 is a mediator of m(6)A-dependent nuclear RNA processing events. Cell 162, 1299-1308 (2015).

69. Han, M. et al. Exosome-transmitted miR-567 reverses trastuzumab resistance by inhibiting ATG5 in breast cancer. Cell Death Dis. 11, 43 (2020).

70. Emens, L. A. The dawn of immunotherapy for breast cancer. Clin. Adv. Hematol. Oncol. 17, 332-335 (2019).

71. Barroso-Sousa, R. et al. Prevalence and mutational determinants of high tumor mutation burden in breast cancer. Ann. Oncol. 31, 387-394 (2020).

72. Michel, L. L. et al. Immune checkpoint blockade in patients with triplenegative breast cancer. Target. Oncol. 15, 415-428 (2020).

73. Chen, G. et al. Exosomal PD-L1 contributes to immunosuppression and is associated with anti-PD-1 response. Nature 560, 382-386 (2018).

74. Poggio, M. et al. Suppression of exosomal PD-L1 induces systemic anti-tumor immunity and memory. Cell 177, 414.e13-427.e13 (2019). 
75. Soliman, H., Khalil, F. \& Antonia, S. PD-L1 expression is increased in a subset of basal type breast cancer cells. PLOS ONE 9, e88557 (2014).

76. Monypenny, J. et al. ALIX regulates tumor-mediated immunosuppression by controlling EGFR activity and PD-L1 presentation. Cell Rep. 24, 630-641 (2018).

77. Wen, S. W. et al. The biodistribution and immune suppressive effects of breast cancer-derived exosomes. Cancer Res. 76, 6816-6827 (2016).

78. Zhang, H. G. et al. Curcumin reverses breast tumor exosomes mediated immune suppression of NK cell tumor cytotoxicity. Biochim. Biophys. Acta 1773, 1116-1123 (2007).

79. Rong, L., Li, R., Li, S. \& Luo, R. Immunosuppression of breast cancer cells mediated by transforming growth factor- $\beta$ in exosomes from cancer cells. Oncol. Lett. 11, 500-504 (2016).

80. Biswas, S. et al. Exosomes produced by mesenchymal stem cells drive differentiation of myeloid cells into immunosuppressive M2-polarized macrophages in breast cancer. J. Immunol. 203, 3447-3460 (2019).

81. Fujiwara, T. et al. Carcinogenic epithelial-mesenchymal transition initiated by oral cancer exosomes is inhibited by anti-EGFR antibody cetuximab. Oral Oncol. 86, 251-257 (2018).

82. Fujiwara, T. et al. Anti-EGFR antibody cetuximab is secreted by oral squamous cell carcinoma and alters EGF-driven mesenchymal transition. Biochem. Biophys. Res. Commun. 503, 1267-1272 (2018).

83. Eguchi, T. et al. Organoids with cancer stem cell-like properties secrete exosomes and HSP90 in a 3D nanoenvironment. PLOS ONE 13, e0191109 (2018)

84. Hu, Y. B. et al. Exosomal Wnt-induced dedifferentiation of colorectal cancer cells contributes to chemotherapy resistance. Oncogene 38, 1951-1965 (2019).

85. Eguchi, T., Taha, E. A., Calderwood, S. K. \& Ono, K. A novel model of cancer drug resistance: oncosomal release of cytotoxic and antibody-based drugs. Biology 9, 47 (2020).

86. Bill, R. \& Christofori, G. The relevance of EMT in breast cancer metastasis: correlation or causality? FEBS Lett. 589, 1577-1587 (2015).

87. Donnarumma, E. et al. Cancer-associated fibroblasts release exosomal microRNAs that dictate an aggressive phenotype in breast cancer. Oncotarget 8, 19592-19608 (2017).

88. Bigagli, E., Cinci, L., D'Ambrosio, M. \& Luceri, C. Transcriptomic characterization, chemosensitivity and regulatory effects of exosomes in spontaneous EMT/MET transitions of breast cancer cells. Cancer Genomics Proteomics $\mathbf{1 6}$ 163-173 (2019).

89. Qin, W. et al. Exosomes in human breast milk promote EMT. Clin. Cancer Res. 22, 4517-4524 (2016).

90. Santos, J. C. et al. Exosome-mediated breast cancer chemoresistance via miR155 transfer. Sci. Rep. 8, 829 (2018)

91. Liu, F., Kong, X., LV, L. \& Gao, J. TGF- $\beta 1$ acts through miR-155 to downregulate TP53INP1 in promoting epithelial-mesenchymal transition and cancer stem cell phenotypes. Cancer Lett. 359, 288-298 (2015).

92. Bai, X., Ni, J., Beretov, J., Graham, P. \& Li, Y. Cancer stem cell in breast cancer therapeutic resistance. Cancer Treat. Rev. 69, 152-163 (2018).

93. Shen, M. et al. Chemotherapy-induced extracellular vesicle miRNAs promote breast cancer stemness by targeting ONECUT2. Cancer Res. 79, 3608-3621 (2019).

94. $\mathrm{Wu}, \mathrm{T}$. \& Dai, Y. Tumor microenvironment and therapeutic response. Cancer Lett. 387, 61-68 (2017).

95. Liu, Q., Peng, F. \& Chen, J. The role of exosomal microRNAs in the tumor microenvironment of breast cancer. Int. J. Mol. Sci. 20, 3884 (2019).

96. Milane, L., Singh, A., Mattheolabakis, G., Suresh, M. \& Amiji, M. M. Exosome mediated communication within the tumor microenvironment. J. Control. Release 219, 278-294 (2015).

97. Lin, T. et al. Synergy of tumor microenvironment remodeling and autophagy inhibition to sensitize radiation for bladder cancer treatment. Theranostics 10 7683-7696 (2020).

98. Xue, M. et al. Hypoxic exosomes facilitate bladder tumor growth and development through transferring long non-coding RNA-UCA1. Mol. Cancer 16, 143 (2017)

99. Logozzi, M., Spugnini, E., Mizzoni, D., Di Raimo, R. \& Fais, S. Extracellular acidity and increased exosome release as key phenotypes of malignant tumors. Cancer Metastasis Rev. 38, 93-101 (2019).

100. Hedlund, M., Nagaeva, O., Kargl, D., Baranov, V. \& Mincheva-Nilsson, L. Thermal- and oxidative stress causes enhanced release of NKG2D ligand-bearing immunosuppressive exosomes in leukemia/lymphoma T and B cells. PLoS ONE 6, e16899 (2011).

101. Kumar, A. \& Deep, G. Hypoxia in tumor microenvironment regulates exosome biogenesis: Molecular mechanisms and translational opportunities. Cancer Lett. 479, 23-30 (2020).

102. Boelens, M. C. et al. Exosome transfer from stromal to breast cancer cells regulates therapy resistance pathways. Cell 159, 499-513 (2014).

103. Gomi, H., Mori, K., Itohara, S. \& Izumi, T. Rab27b is expressed in a wide range of exocytic cells and involved in the delivery of secretory granules near the plasma membrane. Mol. Biol. Cell 18, 4377-4386 (2007).

104. Nabet, B. Y. et al. Exosome RNA unshielding couples stromal activation to pattern recognition receptor signaling in cancer. Cell 170, 352.e13-366.e13 (2017).

105. Bliss, S. A. et al. Mesenchymal stem cell-derived exosomes stimulate cycling quiescence and early breast cancer dormancy in bone marrow. Cancer Res. 76, 5832-5844 (2016).

106. Bovy, N. et al. Endothelial exosomes contribute to the antitumor response during breast cancer neoadjuvant chemotherapy via microRNA transfer. Oncotarget 6, 10253-10266 (2015).

107. Whiteside, T. L. Exosomes and tumor-mediated immune suppression. J. Clin. Investig. 126, 1216-1223 (2016).

108. Amy, M. Braden, Rachel, V. Stankowski, Jessica, M. Engel \& Adedayo, A. Onitilo Breast cancer biomarkers: risk assessment, diagnosis, prognosis, prediction of treatment efficacy and toxicity, and recurrence. Curr. Pharm. Des. 20, 4879-4898 (2014).

109. Bardelli, A. \& Pantel, K. Liquid biopsies, what we do not know (yet). Cancer Cell 31, 172-179 (2017)

110. Campos-Carrillo, A. et al. Circulating tumor DNA as an early cancer detection tool. Pharmacol. Ther. 207, 107458 (2020).

111. Nazri, H. M. et al. Characterization of exosomes in peritoneal fluid of endometriosis patients. Fertil. Steril. 113, 364.e2-373.e2 (2020).

112. Vanhie, A., Tomassetti, C. \& D'Hooghe, T. M. Peritoneal fluid exosomes as potential biomarkers for endometriosis: mind and bridge the gap between innovation and validation/development into benefit for patients. Fertil. Steril. 113, 326-327 (2020).

113. O'Neill, C. P., Gilligan, K. E. \& Dwyer, R. M. Role of extracellular vesicles (EVs) in cell stress response and resistance to cancer therapy. Cancers 11, 136 (2019).

114. Tang, S. et al. Overexpression of serum exosomal HOTAIR is correlated with poor survival and poor response to chemotherapy in breast cancer patients. J. Biosci. 44, 37 (2019).

115. Stevic, I. et al. Specific microRNA signatures in exosomes of triple-negative and HER2-positive breast cancer patients undergoing neoadjuvant therapy within the GeparSixto trial. BMC Med. 16, 179 (2018).

116. Doyle, L. M. \& Wang, M. Z. Overview of extracellular vesicles, their origin, composition, purpose, and methods for exosome isolation and analysis. Cells 8, 727 (2019).

117. Garcia-Cordero, J. L. \& Maerkl, S. J. Microfluidic systems for cancer diagnostics. Curr. Opin. Biotechnol. 65, 37-44 (2019).

118. LeBleu, V. S. \& Kalluri, R. Exosomes as a multicomponent biomarker platform in cancer. Trends Cancer 6, 767-774 (2020).

119. Meldolesi, J. Exosomes and ectosomes in intercellular communication. Curr. Biol. 28, R435-R444 (2018).

120. Hoshino, A. et al. Extracellular vesicle and particle biomarkers define multiple human cancers. Cell 182, 1044.e18-1061.e18 (2020).

121. Datta, A. et al. High-throughput screening identified selective inhibitors of exosome biogenesis and secretion: a drug repurposing strategy for advanced cancer. Sci. Rep. 8, 8161 (2018).

122. Sun, L. et al. Regulation of RAB22A by mir-193b inhibits breast cancer growth and metastasis mediated by exosomes. Int. J. Oncol. 53, 2705-2714 (2018).

123. Koch, R. et al. Nuclear trapping through inhibition of exosomal export by indomethacin increases cytostatic efficacy of doxorubicin and pixantrone Clin. Cancer Res. 22, 395-404 (2016).

124. Kong, J. N. et al. Guggulsterone and bexarotene induce secretion of exosome-associated breast cancer resistance protein and reduce doxorubicin resistance in MDA-MB-231 cells. Int. J. Cancer 137 1610-1620 (2015).

125. Marleau, A. M., Chen, C. S., Joyce, J. A. \& Tullis, R. H. Exosome removal as a therapeutic adjuvant in cancer. J. Transl. Med. 10, 134 (2012).

126. Luan, X. et al. Engineering exosomes as refined biological nanoplatforms for drug delivery. Acta Pharmacol. Sin. 38, 754-763 (2017). 
127. Li, S. et al. Engineering macrophage-derived exosomes for targeted chemotherapy of triple-negative breast cancer. Nanoscale 12, 10854-10862 (2020).

128. Naseri, Z., Oskuee, R. K., Jaafari, M. R. \& Forouzandeh Moghadam, M. Exosomemediated delivery of functionally active miRNA-142-3p inhibitor reduces tumorigenicity of breast cancer in vitro and in vivo. Int. J. Nanomed. 13 7727-7747 (2018).

129. Aqil, F., Munagala, R., Jeyabalan, J., Agrawal, A. K. \& Gupta, R. Exosomes for the enhanced tissue bioavailability and efficacy of curcumin. AAPS J. 19, 1691-1702 (2017).
130. Wolfers, J. et al. Tumor-derived exosomes are a source of shared tumor rejection antigens for CTL cross-priming. Nat. Med. 7, 297-303 (2001).

131. Escudier, B. et al. Vaccination of metastatic melanoma patients with autologous dendritic cell (DC) derived-exosomes: results of thefirst phase I clinical trial. J. Transl. Med. 3, 10 (2005).

132. Li, R., Chibbar, R. \& Xiang, J. Novel EXO-T vaccine using polyclonal CD4(+) $T$ cells armed with HER2-specific exosomes for HER2-positive breast cancer. Onco Targets Ther. 11, 7089-7093 (2018). 